\title{
ASSOCIATION BETWEEN THE ADAPTIVE DIAGNOSIS, THE INDICATORS OF CLINICAL EVOLUTION AND THE OBJECT RELATIONS TEST IN PATIENTS INFECTED BY HIV-1, SICK OR NOT
}

THESIS. N. Silva Filho submitted this thesis for his Doctorate in Tropical Diseases at Botucatu School of Medicine, São Paulo State University, UNESP, Botucatu, São Paulo, Brazil, 2003.

Advisor: Professor Lenice do Rosário de Souza

\begin{abstract}
Thirty-one individuals (14 men and 17 women) infected by HIV-1, sick or not, were evaluated at the Special Outpatient Clinic of the Tropical Diseases Area, Department of Tropical Diseases and Imaging Diagnosis, Botucatu School of Medicine, UNESP, São Paulo. Sixteen patients had two psychological evaluations in different moments. For these evaluations, the Phillipson Object Relations Test (TRO) and the Operational Adaptive Diagnostic Scale (EDAO) were used, being isolated the internal and external, positive and negative factors: quality of the familial relationship during childhood, depression, irritability, impulsiveness, religious fanaticism, pregnancy and/or precocious sexuality, prevalence of the mind's psychotic operation, suicidal tendency, and abusive consumption of illicit drugs. The epidemic evaluation took into consideration: sexual option, qualifications, marital status, religion, occupation, living alone or with other people, form of contracting the infection, existence of spouses and children with HIV infection, and place of residence. The clinical evaluation considered the plasmatic viral load, the counting of lymphocytes T CD4 ${ }^{+}$and $\mathrm{CD}^{+}$, the number of hospital admissions, development or not of symptoms compatible with AIDS, presence of neurological diseases, adhesion and antiretroviral (ARV) medication type, time of diagnosis, phase of life (adolescence or adulthood) in which the infection was acquired. It was observed that $90.32 \%$ of the individuals were in the productive age, and a significant part of the sample (29.03\%) developed the infection in the adolescence, being almost the totality by sexual contact, many times accompanied by pregnancy and/or an undesired paternity. After contracting the infection, some individuals became user or continued to use illicit drugs, an additional difficulty in life conditions. The ones that were infected by injecting drugs use (IDU) had already developed AIDS, presented neurological diseases, and needed hospital admission; the evaluation of the adaptive effectiveness indicated severe ineffective adaptation. Prevalence of the mind's psychotic operation was identified in the adaptive effectiveness deterioration, suggesting the presence of pathological organizations as defense. The individuals that presented AIDS,
\end{abstract}


prevalence of the mind's psychotic operation, pregnancy and/or precocious sexuality, impulsiveness, and irritability showed significant deterioration of the adaptive effectiveness among the first psychological evaluations. Patients with suicidal intention and/or severe ineffective adaptation present trend line of the variation of decreasing $\mathrm{CD}^{+}$, decreasing $\mathrm{CD}^{+}$, and high plasmatic viral load. The ones that presented suicidal intention, showed trend line of high plasmatic viral load, and the ones that presented at least more than one evaluation of high plasmatic viral load with trend line of decreasing viral load, and increasing or stable $\mathrm{CD}^{+}$and $\mathrm{CD}^{+}$, showed severe ineffective adaptation. Most of the patients presented chronic depression of the psychotic type. The personality characteristics would be exacerbated, producing symptoms such as depression, irritability, hallucinosis, paranoid delirium, etc. The capacity to face adversities was very limited, as demonstrated by TRO and EDAO. The higher adaptive balance, measured by TRO, would be accompanied of a larger preservation of the immunological system, independently of the resources and difficulties that those individuals would find, day by day, in working with the adversities of the external world, as evaluated by EDAO. Positive correlation was observed between TRO and EDAO, TRO and $\mathrm{CD}^{+}, \mathrm{EDAO}$ and $\mathrm{CD} 8^{+}$. These data suggest that individuals infected by HIV present higher adaptive balance when the adaptive effectiveness is higher. The effects of the pathological organizations were observed in the involvement of the organic sector through the difficulties for "compliance", depression, irritability, forming and maintaining contacts, suicide intention, impulsiveness, and finally, prevalence of the mind's psychotic operation.

KEY WORDS: AIDS, HIV.

\section{CORRESPONDENCE TO:}

N. SILVA FILHO, Departamento de Doenças Tropicais e Diagnóstico por Imagem, Faculdade de Medicina de Botucatu, UNESP, Distrito de Rubião Junior, s/n, 18618-000, Botucatu, SP, Brasil. Phone: 55143811 6212. Email: Isouza@fmb.unesp.br 